\title{
Photothermally Activated Two-Photon Absorption in Ion-Implanted Silicon Quantum Dots in Silica Plates
}

\author{
C. Torres-Torres $\mathbb{D}^{1},{ }^{1}$ J. Bornacelli $\left(\mathbb{D},{ }^{2}\right.$ R. Rangel-Rojo, ${ }^{3}$ J. A. García-Merino, ${ }^{1}$ B. Can-Uc, ${ }^{4}$ \\ L. Tamayo-Rivera, ${ }^{5}$ J. C. Cheang-Wong, ${ }^{2}$ L. Rodríguez-Fernández $\left(\mathbb{D},{ }^{2}\right.$ and A. Oliver ${ }^{2}$ \\ ${ }^{1}$ Sección de Estudios de Posgrado e Investigación, Escuela Superior de Ingeniería Mecánica y Eléctrica Unidad Zacatenco, \\ Instituto Politécnico Nacional, 07738 Ciudad de México, Mexico \\ ${ }^{2}$ Instituto de Física, Universidad Nacional Autónoma de México, 04510 Ciudad de México, Mexico \\ ${ }^{3}$ Departamento de Óptica, Centro de Investigación Científica y de Educación Superior de Ensenada, A.P. 360, \\ 22860 Ensenada, BC, Mexico \\ ${ }^{4}$ Centro de Nanociencias y Nanotecnología, Universidad Nacional Autónoma de México, A.P. 14, 22800 Ensenada, BC, Mexico \\ ${ }^{5}$ Escuela Superior de Apan, Universidad Autónoma del Estado de Hidalgo, 43920 Apan, HGO, Mexico \\ Correspondence should be addressed to C. Torres-Torres; crstorres@yahoo.com.mx
}

Received 16 November 2017; Revised 28 December 2017; Accepted 24 January 2018; Published 20 February 2018

Academic Editor: Sergio Obregón

Copyright (C) 2018 C. Torres-Torres et al. This is an open access article distributed under the Creative Commons Attribution License, which permits unrestricted use, distribution, and reproduction in any medium, provided the original work is properly cited.

\begin{abstract}
The third-order nonlinear infrared and ultraviolet properties exhibited by silicon quantum dots irradiated by ultrashort pulses were studied. The samples were prepared by $1.5 \mathrm{MeV} \mathrm{Si}^{+2}$ ion implantation processes in high-purity silica substrates. Femtosecond $z$-scan measurements conducted at $830 \mathrm{~nm}$ wavelength revealed strong self-focusing effects together with two-photon absorption that can be switched to saturable absorption as a function of the input irradiance. Changes in the main physical mechanism responsible for the picosecond absorptive nonlinearity in the sample were also observed at $355 \mathrm{~nm}$, made possible by the assistance of photothermal phenomena. Ultraviolet self-diffraction explorations allowed us to estimate the Kerr effect of the nanostructures. Potential applications for developing all-optical filtering functions performed by silicon-based nanosystems can be considered.
\end{abstract}

\section{Introduction}

Recent progress in device fabrication opens up fascinating possibilities for the incorporation of advanced nanostructured materials to enhance the performance of all-optical operations [1]. Integrated nanophotonic systems could provide platforms for developing ultracompact signal processing tools and dense configurations for manipulating optical information in the ultrafast regime [2]. The potential to multiplex large data streams by ultrashort laser pulses seems to be a suitable alternative for solving some of the key challenges in several technological applications [3]. Nonlinear optical effects anticipate the all-optical implementation of multifunctional photonic elements that can simultaneously perform various signal processing tasks [4]. In the same direction, distinct computing approaches based on quantum network technologies can be envisioned as essential tools for designing novel optical data communication systems [5].
Silicon-based materials have been employed as the main components for building modern electronic and optoelectronic systems [6]. The dynamics of electronic and photothermal processes emerging from silicon quantum dots (SiQDs) seem to be attractive for the development of extremely small structures that confine the fundamental charge carriers responsible for semiconductive phenomena [7]. Silicon nanostructures exhibit powerful optical and nonlinear optical properties that can be attractive for the development of a new generation of nonlinear photonic devices [8]. In particular, it has been pointed out that excitonic effects associated with SiQDs can be derived by quantum confinement mechanisms, and they could play a critical role in the enhancement of the nonlinear optical response [9].

From these collective excitations related to periodically ordered SiQDs arrays, one can consider the enhancement of nanophotonic properties that could promote superior light 
manipulation techniques at the nanoscale [10]. The involvement of discrete density of states of SiQDs under illumination may originate essential interactions for practical applications such as low-dimensional laser systems [11], single-photon sources [12], photonic waveguide circuits [13], or all-optical switching devices [14].

Optical and electronic transitions associated with highirradiance beams can be also considered to generate absorptive or refractive nonlinearities that potentially play a crucial role for modulating photonic signals [15]. Since the nonlinear optical properties exhibited by SiQDs are drastically dependent on the size and size distribution of nanoparticles in the sample, then also the processing route for their synthesis can significantly influence their optical properties [16]. In this respect, $\mathrm{MeV}$ ion implantation technique allows the versatile design of structured samples with SiQDs following a particular depth profile distribution that can be controlled by the energy, fluence, and angle of the ion beam. Moreover, ion implantation is fully compatible with CMOS technology processing design in actual microelectronics. Then, one of the main advantages of this synthesis method is its potential to create nanosystems that may be attractive for direct applications that require very stable optical and mechanical characteristics.

In view of all of these elements, the topic discussed in this paper mainly presents an analysis of the third-order nonlinear infrared and ultraviolet effects exhibited by ion-implanted SiQDs embedded in silica plates. A modification from ultraviolet saturated absorption to two-photon absorption in the sample was observed by a photothermal action able to demodulate laser signals. A picosecond self-diffraction method was carried out to explore the magnitude of the third-order nonlinear susceptibility tensor of the sample at a $355 \mathrm{~nm}$ wavelength, while a $z$-scan technique was employed to measure infrared femtosecond nonlinear absorption and refraction effects. Within the text we discussed the fascinating nonlinearities that could be engineering in SiQDs by the contribution of cooperative interactions.

\section{Experimental}

2.1. Sample Preparation. The samples were synthesized by using the 3 MV Tandem accelerator (NEC 9SDH-2 Pelletron) facility at the Instituto de Física of the Universidad Nacional Autónoma de México. High-purity silica glass plates $(25 \times 25$ $\times 1 \mathrm{~mm}$ ) with $\mathrm{OH}$ content less than $1 \mathrm{ppm}$ and total impurity content less than $20 \mathrm{ppm}$ were employed as substrates. The room temperature ion implantation was performed by means of $1.5 \mathrm{MeV} \mathrm{Si}^{+2}$ ions at a fluence of $2.5 \times 10^{17}$ ions $/ \mathrm{cm}^{2}$. The Si-implanted samples were then thermally annealed in a reducing atmosphere $\left(50 \% \mathrm{~N}_{2}+50 \% \mathrm{H}_{2}\right)$ at $1100^{\circ} \mathrm{C}$ for 1 hour. A FEI Tecnai F30 Transmission Electron Microscope (FEGTEM $300 \mathrm{kV}$ ) in scanning mode (STEM) with a high angle annular dark field (HAADF) detector ( $Z$-contrast) was used to perform nanoscopic observations on the sample. The characteristic optical absorption spectrum of the studied samples was recorded by using an UV-visible spectrometer (Varian Cary 5000). Nonlinear optical measurements were investigated during different years in different regions of similar samples systematically studied.
2.2. Femtosecond $z$-Scan Explorations. The nonlinear absorption and nonlinear refraction exhibited by the samples with embedded SiQDs were studied by the $z$-scan technique using infrared femtosecond irradiation [17]. Z-scan measurements were undertaken by using a Ti:sapphire laser system with $80 \mathrm{fs}$ pulses at $830 \mathrm{~nm}$ wavelength focused in a beam waist of $47 \mu \mathrm{m} \pm 0.5 \mu \mathrm{m}$, resulting in a peak irradiance of with $48 \mathrm{MW} / \mathrm{cm}^{2}$, and a repetition rate of $94 \mathrm{MHz}$. The transmittance for the open-aperture $z$-scan technique can be described by [17]

$$
T\left(z, \Delta \Phi_{o}\right)=1-\frac{\beta I_{o} L_{\mathrm{eff}}}{2 \sqrt{2}\left(x^{2}+1\right)},
$$

where $\beta$ corresponds to the two-photon absorption coefficient, $L_{\text {eff }}$ is the effective length given by $L_{\text {eff }}=\left(1-e^{-\alpha_{o} L}\right) / \alpha_{o}$ with $L$ the sample length, and $\alpha_{o}$ is the linear absorption coefficient.

On the other hand, for the transmittance of the closedaperture $z$-scan measurements, we considered [17]

$$
T\left(z, \Delta \Phi_{o}\right)=1-\frac{4 \Delta \Phi_{o} x}{\left(x^{2}+9\right)\left(x^{2}+1\right)},
$$

where $x=z / z_{o}, z_{o}=k w_{o}^{2} / 2, k=2 \pi / \lambda, \lambda$ represents the laser wavelength in free space, and the on-axis optical phase shift of the beam can be denoted by $\Delta \Phi_{o}$ as

$$
\Delta \Phi_{o}=k \Delta n_{o} L_{\text {eff }},
$$

the on-axis maximum refractive index change can be represented by $\Delta n_{0}=n_{2} I_{0}$ and related to the nonlinear refractive index, $n_{2}$, and $I_{0}$ that corresponds to the focused peak on-axis irradiance.

2.3. Time-Resolved Optical Kerr Gate Evaluations. Optical Kerr gate $(\mathrm{OKG})$ studies were undertaken in order to see the femtosecond time-resolved behavior of the SiQDs samples [18]. The pulses were provided by a Ti:sapphire laser system emitting a $825 \mathrm{~nm}$ wavelength, $80 \mathrm{fs}$ pulses, and $3 \mathrm{~nJ}$ maximum pulse energy at $94 \mathrm{MHz}$. Pump and probe beams presented an irradiance relation of $15: 1$, vectorial planes of linear polarization making an angle of $45^{\circ}$, and a spot size focused in about $80 \mu \mathrm{m}$. The Kerr signal was recorded by a photodetector acquiring the probe irradiance propagated through an analyzer crossed with respect to the initial polarization of the probe beam. A lock-in amplifier assisted the experiment to detect the frequency related to the probe beam passing through a mechanical chopper. The probe transmittance as a function of the electronically controlled delay time between the pump to probe was measured.

2.4. Picosecond Ultraviolet Irradiation Studies. The singlebeam transmittance of the sample irradiated by the third harmonic (355 nm) emerging from a Nd:YAG laser system (EKSPLA) with pulse duration of 25 ps was explored. Moreover, a degenerated two-wave mixing experiment with the same laser source was carried out to identify the magnitude of the third-order nonlinear ultraviolet response exhibited by 
the sample. The incident probe and pump beams at $355 \mathrm{~nm}$ wavelength were linearly polarized and adjusted to present an irradiance relation of $1: 10$. These two laser beams, focusing on the sample with a geometric angle of about $10^{\circ}$, a spot size $\sim 0.2 \mathrm{~mm}$, and a total incident energy of $55 \mu \mathrm{J}$, were analyzed at two different repetition rates, $1 \mathrm{~Hz}$ and $10 \mathrm{~Hz}$ repetition rates, respectively. The polarization of the probe beam was fixed, while the polarization of the pump beam was rotated with a half-wave plate. The self-diffracted irradiances were acquired by using a high speed Ga photodetector (DET 25K Thorlabs).

The expressions for describing the approximated amplitudes of the transmitted fields are [19]

$$
\begin{aligned}
& E_{1 \pm}(z)=\left[J_{0}\left(\Psi_{ \pm}^{(1)}\right) E_{1 \pm}+i J_{1}\left(\Psi_{ \pm}^{(1)}\right) E_{2 \pm}\right] \\
& \cdot \exp \left(-i \Psi_{ \pm}^{(0)}-\frac{\alpha(I) z}{2}\right), \\
& E_{2 \pm}(z)=\left[J_{0}\left(\Psi_{ \pm}^{(1)}\right) E_{2 \pm}-i J_{1}\left(\Psi_{ \pm}^{(1)}\right) E_{1 \pm}\right] \\
& \cdot \exp \left(-i \Psi_{ \pm}^{(0)}-\frac{\alpha(I) z}{2}\right), \\
& E_{3 \pm}(z)=\left[i J_{1}\left(\Psi_{ \pm}^{(1)}\right) E_{1 \pm}-J_{2}\left(\Psi_{ \pm}^{(1)}\right) E_{2 \pm}\right] \\
& \cdot \exp \left(-i \Psi_{ \pm}^{(0)}-\frac{\alpha(I) z}{2}\right), \\
& E_{4 \pm}(z)=\left[-i J_{1}\left(\Psi_{ \pm}^{(1)}\right) E_{2 \pm}-J_{2}\left(\Psi_{ \pm}^{(1)}\right) E_{1 \pm}\right] \\
& \cdot \exp \left(-i \Psi_{ \pm}^{(0)}-\frac{\alpha(I) z}{2}\right),
\end{aligned}
$$

where $E_{1 \pm}(z)$ and $E_{2 \pm}(z)$ are the complex amplitudes associated with the circularly polarized components of the transmitted waves beams; $E_{3 \pm}(z)$ and $E_{4 \pm}(z)$ are the amplitudes of the self-diffracted waves; $\alpha(I)$ is the irradiance dependent absorption coefficient; $J_{m}\left(\Psi_{ \pm}^{(1)}\right)$ stands for the Bessel function of order $m$; $z$ is the thickness of the nonlinear media; and $\Psi_{ \pm}^{(0)}$ and $\Psi_{ \pm}^{(1)}$ are the nonlinear phase changes.

2.5. Photothermal Analysis. The photothermal response exhibited by the sample was measured by a Wheatstone bridge circuit with a thermistor as a sensor of temperature. The photothermal experiment was conducted with a $355 \mathrm{~nm}$ focused beam featuring $25 \mathrm{ps}$ pulse duration and $10 \mathrm{~Hz}$ repetition rate. The change in temperature $(\Delta T)$ was induced by $650 \mu \mathrm{J}$ laser excitation for 10 seconds in a spot area of $47.12 \mathrm{~mm}^{2}$. The temporal thermal response of the SiQDs sample was acquired by an infrared sensor with a resolution of $0.01^{\circ} \mathrm{C}$. The signal was conditioned with a low noise amplifier and a 17-bit high-resolution analog-digital converter. The data were collected into a PC by an 8-bit Atmel microcontroller. In order to fit the experimental data, the numerical solution of the $2 \mathrm{D}$ heat equation was considered,

$$
\frac{\partial T(x, y, t)}{\partial t}=\frac{k}{c_{p} \rho} \nabla^{2} T(x, y, t)+\frac{1}{c_{p} \rho} P(x, y, t),
$$

where $t$ represents the time, $x$ and $y$ represent the spatial coordinates transverse to the propagation of the beam, $k$

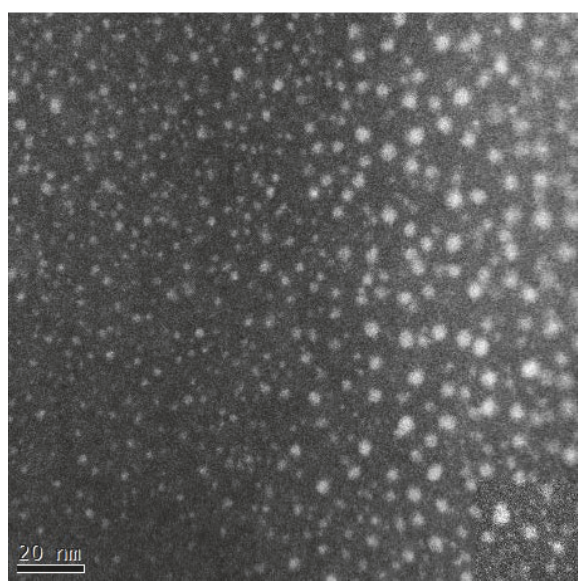

FIGURE 1: Panoramic STEM micrograph of a representative sample with embedded SiQDs.

represents the thermal conductivity, $c_{p}$ represents the specific heat, $\rho$ represents the density, and $P$ represents the Fourier series of the laser pumping. The parameters to approach the function were thickness $1 \mathrm{~mm}$ of the silica substrate and $400 \mathrm{~nm}$ for the FWHM of the implanted Si ions estimated by SRIM simulation [20]. The absorption coefficient is ten times smaller in pure silica that in the nanostructured samples with embedded SiQDs according spectroscopic measurements. Consequently, we can assume that thermal transport was mainly induced by the phonon dispersion on the SiQDs excited by the ultraviolet irradiation. Moreover, we reproduced the experiment in pristine silica plates to corroborate that no change in temperature was achieved under the same laboratory conditions.

2.6. Low-Pass Filter by Nonlinear Ultraviolet Absorption Effects in SiQDs. Ultraviolet picosecond pulses provided by our $\mathrm{Nd}$ :YAG system with two different repetition rates, c.a. $10 \mathrm{~Hz}$ and $1 \mathrm{~Hz}$, were employed as a laser signal featuring a pulsedensity-modulation (PDM). Here, PDM signals correspond to binary signals distinguished by their characteristic frequency, $f_{0}=10 \mathrm{~Hz}$ and $f_{1}=1 \mathrm{~Hz}$, for representing the digital 0 or 1 value, respectively. The demodulation of the PDM signals was obtained through the nonlinear transmittance of the ionimplanted SiQDs sample behaving like a low-pass filter.

\section{Results and Discussions}

Figure 1 shows a STEM image of our samples where the white points in the micrograph represent the SiQDs. The estimated average size of the SiQDs was approximately $6 \mathrm{~nm}$ $\pm 2.5 \mathrm{~nm}$. SRIM calculations indicated that the FWHM of the Si depth profile distribution is $\sim 400 \mathrm{~nm}$ with a maximum peak concentration located at about $1700 \mathrm{~nm}$ below the surface of the silica substrate.

In Figure 2 the comparative UV-Vis spectra for the silica sample with embedded SiQDs and the pristine silica are illustrated. For the SiQDs sample, a well-defined absorption edge can be visualized. Reproducibility in the fabrication of 


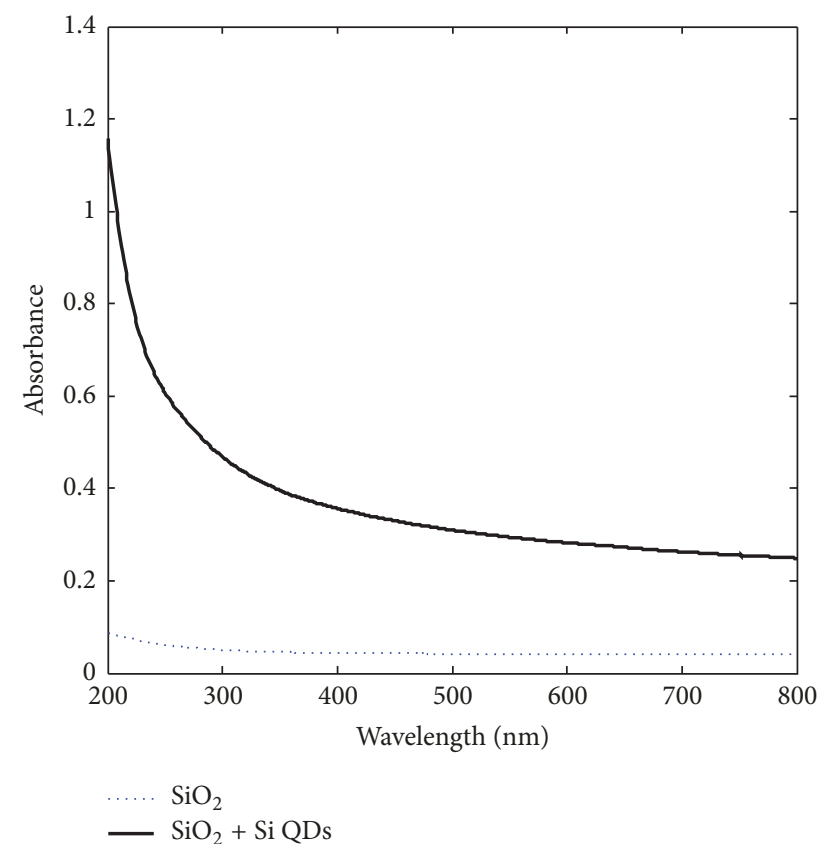

FIGURE 2: Optical absorption spectra of the sample with embedded SiQDs and the pristine silica.

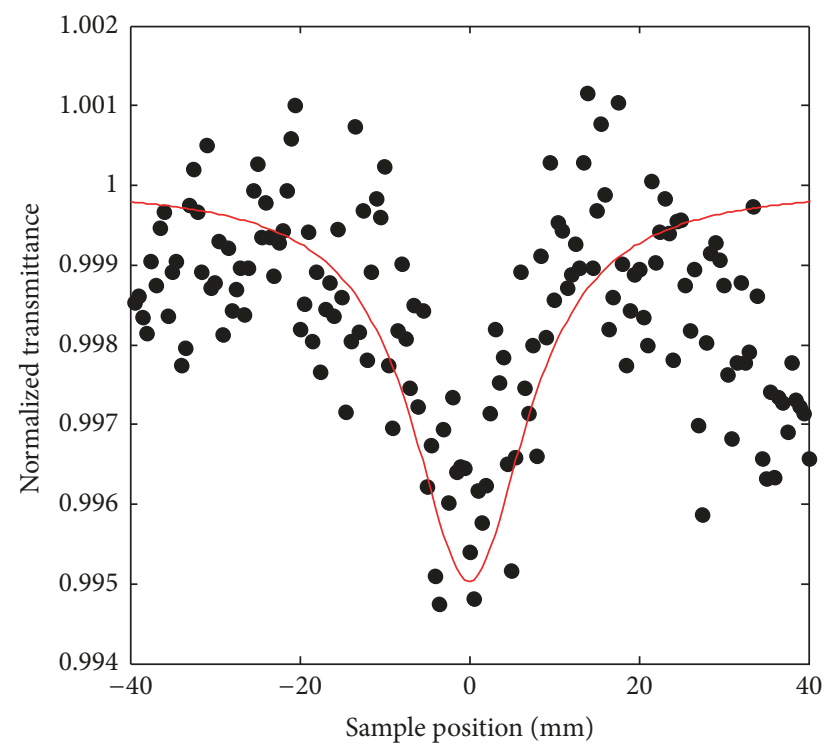

Figure 3: Femtosecond open-aperture $z$-scan data at $830 \mathrm{~nm}$.

the sample was guaranteed by SEM and UV-Vis spectroscopic measurements. Crystallinity of the SiQDs has been previously verified by photoluminescence analysis of comparative samples prepared by ion implantation [10].

Figure 3 describes the open-aperture $z$-scan data ( $80 \mathrm{fs}$ at $830 \mathrm{~nm}$ excitation wavelength) resolving a two-photon absorption effect; that is, a diminishing transmittance at the focal plane can be seen. The fit in the plot was computed by using (1) and the estimated $\beta=1.6 \times 10^{-7} \mathrm{~m} / \mathrm{W}$ magnitude.

The closed-aperture $z$-scan experiments (Figure 4 ) denote a positive nonlinear refractive index $n_{2}$ for the fs

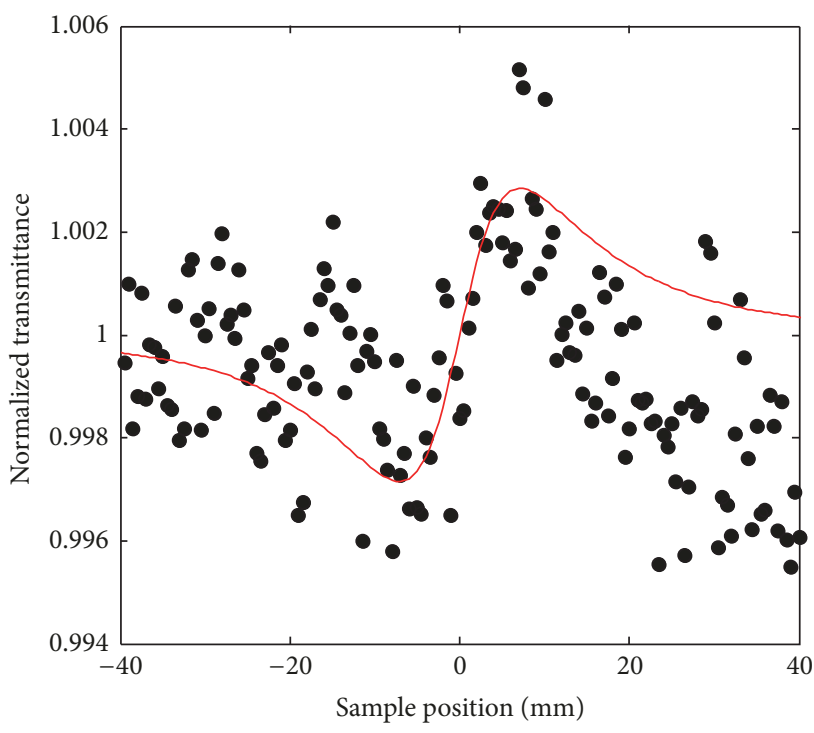

FIgURE 4: Femtosecond closed-aperture $z$-scan data at $830 \mathrm{~nm}$.

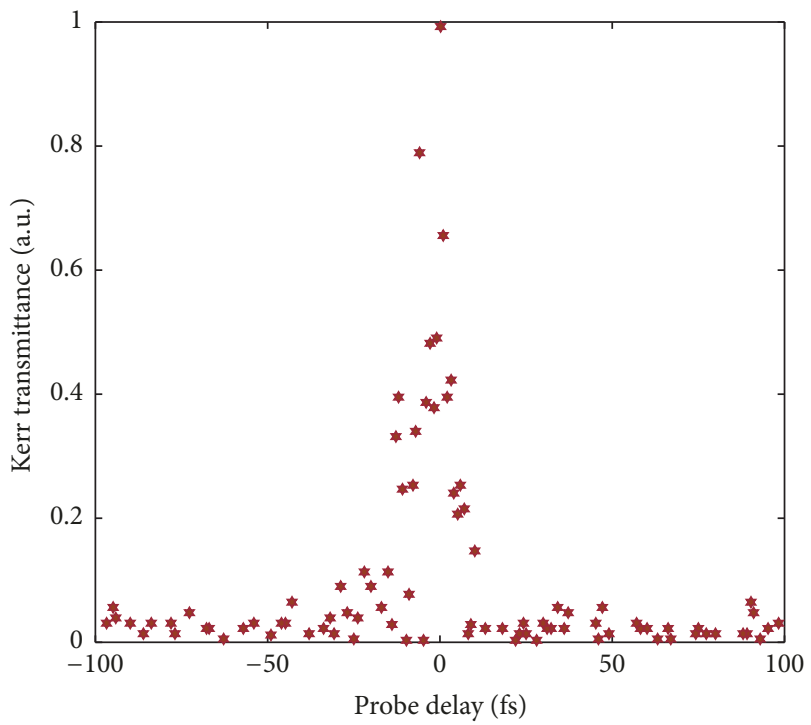

FIGURE 5: Time-resolved OKG signal for the SiQDs samples studied.

pulses. The value $n_{2}=2.11 \times 10^{-14} \mathrm{~m}^{2} / \mathrm{W}$ was obtained by the fitting performed by using (2).

Figure 5 demonstrates the ultrafast time-resolved OKG measurements collected for the SiQDs excited by infrared fs pulses. The data demonstrates a nonlinear Kerr response that raises and decays during the femtosecond pulse duration, which can be associated with an ultrafast purely electronic optical behavior exhibited by the nanostructures.

Ablation was not observed in the sample during femtosecond experiments up to the maximum $3 \mathrm{~nJ}$ energy available, focused in a beam waist of approximately $47 \mu \mathrm{m}$. Furthermore, picosecond ultraviolet damage of the sample was observed with $0.2 \mathrm{~J} / \mathrm{cm}^{2}$ pulses. This ablation threshold magnitude corresponds to a single shot focused on an area 
TABLE 1: Nonlinear infrared and ultraviolet coefficients estimated.

\begin{tabular}{lccc}
\hline Laser irradiation & $\beta(\mathrm{m} / \mathrm{W})$ & $n_{2}\left(\mathrm{~m}^{2} / \mathrm{W}\right)$ & $\left|\chi^{(3)}\right|(\mathrm{esu})$ \\
\hline $830 \mathrm{~nm}, 80 \mathrm{fs}, 94 \mathrm{MHz}$ & $1.6 \times 10^{-7}$ & $2.11 \times 10^{-14}$ & $1.8 \times 10^{-8}$ \\
$355 \mathrm{~nm}, 25 \mathrm{ps}, 10 \mathrm{~Hz}$ & $2.1 \times 10^{-9}$ & $1.21 \times 10^{-16}$ & $1.05 \times 10^{-10}$ \\
$355 \mathrm{~nm}, 25 \mathrm{ps}, 1 \mathrm{~Hz}$ & $-2.4 \times 10^{-9}$ & & \\
\hline
\end{tabular}

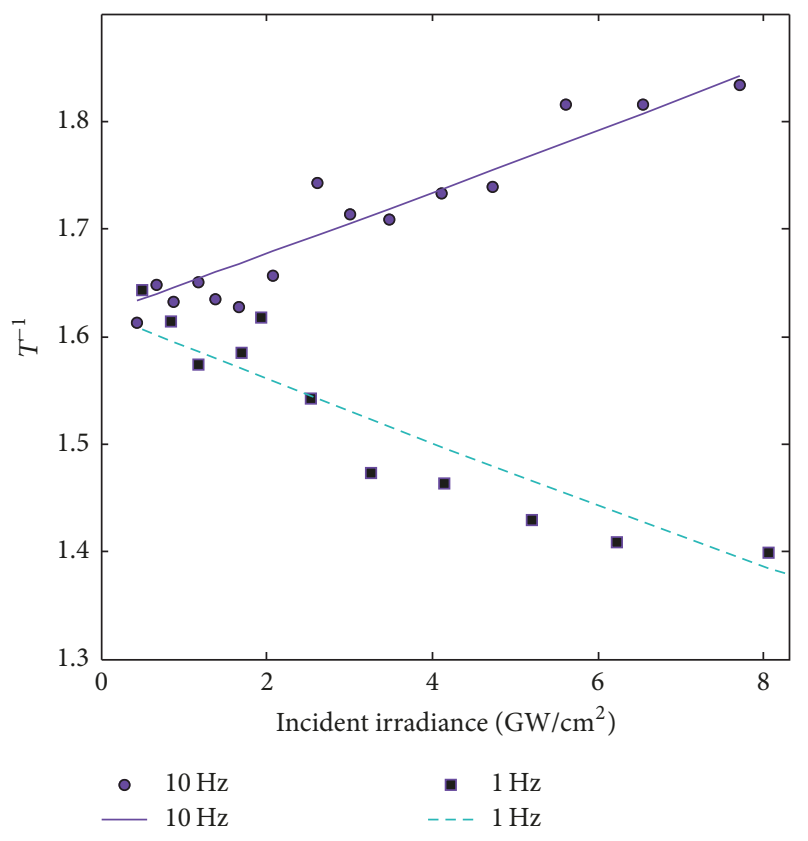

FIGURE 6: Contrast in the experimental inverse of the transmittance as a function of the incident irradiance at $355 \mathrm{~nm}$ and $25 \mathrm{ps}$ in propagation through the SiQDs sample studied at two different repetition rates: $1 \mathrm{~Hz}$ or $10 \mathrm{~Hz}$.

with $0.1 \mathrm{~mm}$ radius in the waist, $355 \mathrm{~nm}$ wavelength, and $65 \mu \mathrm{J}$.

The ultraviolet transmittance of the sample, $T$, was measured as a function of the input irradiance, $I_{o}$. The results that correspond to $T^{-1}$ versus $I_{o}$ are plotted in Figure 6. An error bar of $\pm 10 \%$ was estimated for the irradiance data. The dots in the graph correspond to experimental data and the lines are related to numerical fits adjusted by considering the total irradiance of the incident beam as $I_{o}$ and the transmitted irradiance $I(L)$ as

$$
I(L)=\frac{I_{o} \exp \left(-\alpha_{o} L\right)}{1+\beta I_{o} L_{\mathrm{eff}}} .
$$

Expression (6) clearly shows that the inverse of the transmittance $\left(T^{-1}\right)$ is a linear function of input irradiance, with the slope related to the two-photon absorption coefficient $\beta$. Figure 6 shows the experimental results plotted as $T^{-1}$ versus $I_{0}$, together to linear fits to the data at the two repetition rates employed. A clear divergence in the behavior of the inverse of the transmittance as a function on the irradiance at two different repetition rates can be observed in Figure 6. By using a repetition rate of $1 \mathrm{~Hz}$, induced transparency (saturable absorption) is obtained, while the increase in the

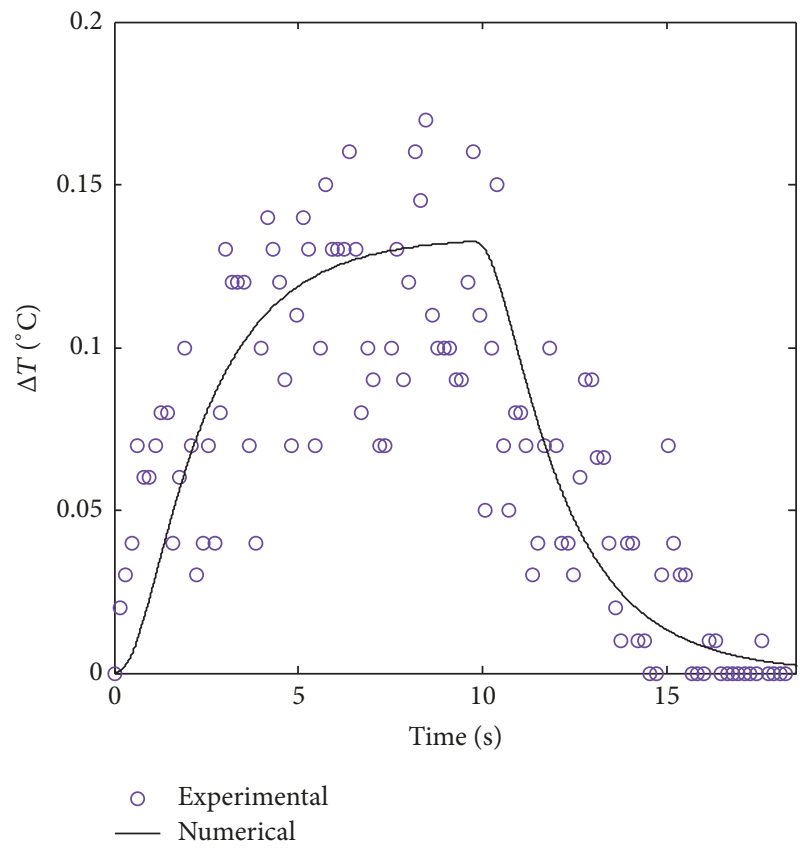

FIGURE 7: Experimental and numerical photothermal response exhibited by the SiQDs sample exposed to picosecond laser pulsed irradiation at $355 \mathrm{~nm}$ wavelength.

repetition rate to $10 \mathrm{~Hz}$ notably produces the signature of a multiphotonic absorption (induced absorption) process in the sample. The fit to the data yields $\beta=-2.4 \times 10^{-9} \mathrm{~m} / \mathrm{W}$ for the $1 \mathrm{~Hz}$ data set and $\beta=2.1 \times 10^{-9} \mathrm{~m} / \mathrm{W}$ for the $10 \mathrm{~Hz}$ set, and the values are contained in Table 1 . We confirmed the possibility of controlling the slope of the nonlinear transmittance with the modification of the repetition rate from $1 \mathrm{~Hz}$ to $10 \mathrm{~Hz}$. The numerical solution of the $2 \mathrm{D}$ heat equation expressed in (5) and experimental results showed in Figure 7 demonstrated that a photothermal process takes place in the picosecond experiments when the repetition rate is increased. Apparently, the change in dynamics of the energy deposited gives rise to a remarkable modification in the nonlinear ultraviolet absorbance transitions. Usually, in order that saturable absorption can be present, there should be an absorption resonance close to the excitation wavelength as it corresponds to our experiments at a $355 \mathrm{~nm}$. Subsequently, we inferred that this change in nonlinear transmittance in the sample can be associated with a variation in the nonlinear ultraviolet absorbance resonance. This last inference implies a potential tuning of the two-photon absorption effect. Auger recombination and the temporal dynamics related to the photonic interaction in the sample may receive an important energy contribution that could modify the valence band 
electron density, automatically affecting the absorption as a function on the irradiance. The irradiance magnitude used in this work is enough to increase the probability to get two pairs of e-h (excitons) per SiQDs. Auger recombination in SiQDs, due to Coulomb interactions between carriers, is very fast (about $10 \mathrm{ps}$ to $100 \mathrm{~ns}$ ) and it limits the number of excitons per SiQDs to a maximum of two. Then, as the energy of the incident pulse increases, it is expected that a larger number of SiQDs with two excitons also increase until a saturated behavior is reached; this can explain the induced transparency observed in Figures 3 and 6 [21]. On the other hand, if the repetition rate of laser pulses is increased, two effects could take place: intraband absorption of electrons in the excited state [22] or biexciton emission [23] and both effects can explain the reduced transmittance of the SiQDs observed in this work. Biexciton deexcitation is a very inefficient process, but it is enhanced for higher temperatures and stronger laser intensities. The decay time for biexciton emission is of the order of Auger recombination, and it corresponds to a decay time of the order of $30 \mathrm{~ns}$ for SiQDs. The lifetime related to the release of excited states in ion-implanted SiQDs is governed by an exponential beta function that results from the interaction between the electrical fields emitted by different SiQDs. This situation typically originates stored excitation energy as slow as $100 \mu \mathrm{s}$; comparative experimental fluorescence studies are in good agreement with this asseveration [10]. Taking into account the lifetime of excited transitions in SiQDs, differences in the nonlinear optical behavior exhibited by Figure 6 can receive an important influence from intraband and Auger effects processes which are more likely to occur for $10 \mathrm{~Hz}$ rather than $1 \mathrm{~Hz}$ pulse rate. On the other hand, intraband absorption processes can result after photon excitation into the conduction band of the SiQDs. Then electrons decay quickly (subpicoseconds) to the lowest energy state within the conduction band. At the same time, this excited electron may also make an electronic transition to higher energy level by absorbing another incoming photon [22]. Therefore, the increase in absorbed energy from the laser pulses reduces the transmittance of the sample.

In order to experimentally identify if a photothermal process would be responsible for our observations, the sample temperature was monitored. Using a single-beam irradiation with a $1 \mathrm{~Hz}$ repetition rate no temperature change was observed up to the ablation threshold levels; however a noticeable induced temperature change was recorded with a $10 \mathrm{~Hz}$ repetition rate. Figure 7 shows the induced temperature change in the sample when ultraviolet picosecond pulses irradiated the sample with a $10 \mathrm{~Hz}$ repetition rate. This experiment corresponds to an irradiation time of 10 seconds regarding the fact that the results were compared for repetition rates of $1 \mathrm{~Hz}$ and $10 \mathrm{~Hz}$.

The change in temperature described by Figure 7 concerns 10 seconds of optical irradiation followed by the evolution of the heat dissipation with a 1/e decay close to 1 second. A significant temperature variation could be responsible for nonlinear refraction and for that reason the third-order nonlinear ultraviolet response of the sample was explored by a self-diffraction technique [19]. The optical Kerr effect can

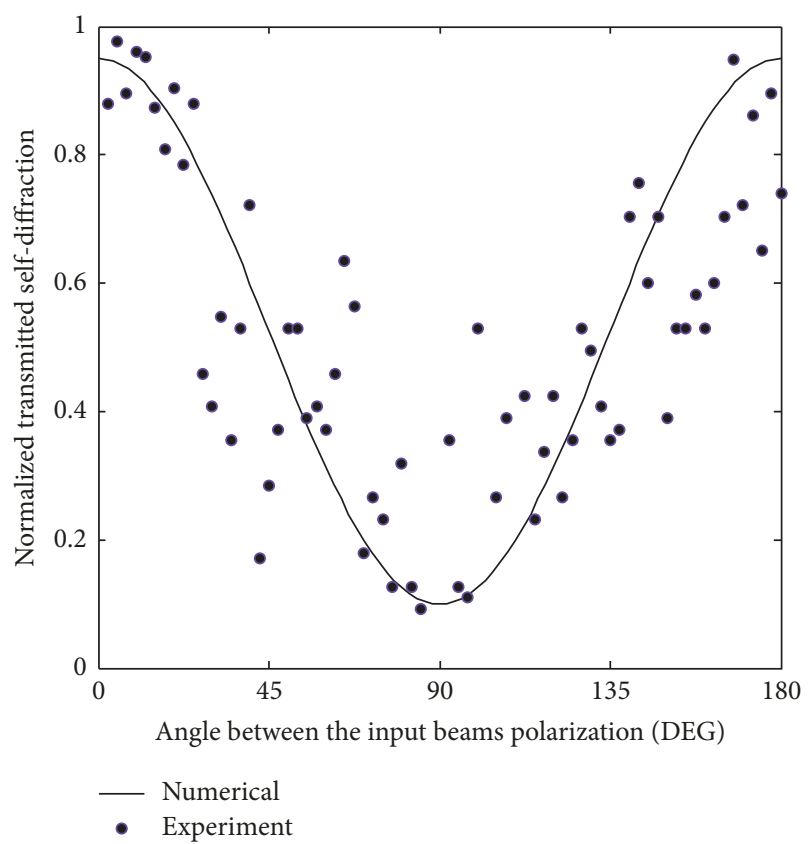

FIGURE 8: Normalized probe transmittance as a function of the angle of polarization between the interacting beams in the SiQDs sample studied.

be derived from different physical mechanisms of nonlinear refractive index. A thermal process can be associated with a thermooptic coefficient that may be important for the modification of self-diffraction phenomena obtained by twowave mixing experiments. The rotation of the angle between the planes of polarization of two incident beams in the sample allows analyzing the vectorial self-diffracted behavior. In Figure 8 representative self-diffraction signals obtained by a two-wave mixing experiment are shown; the repetition rate of the laser excitation was $10 \mathrm{~Hz}$ in this case. The nonzero value of the self-diffracted signal obtained by orthogonal polarization of the incident beams indicates an electronic mechanism as the main responsible for the optical Kerr effect induced in the sample by third-order nonlinearities [23]. The self-diffraction system was calibrated by using a well-known $\mathrm{CS}_{2}$ standard sample. An error bar of $\pm 10 \%$ was estimated for the experimental data. Using (4), $\left|\chi^{(3)}\right|=1.05 \times 10^{-10}$ esu value was estimated, corresponding to the SiQDs sample studied in this picosecond regime at $355 \mathrm{~nm}$.

We considered the relationship between the nonlinear refractive index $n_{2}$ and $\beta$ with $\chi^{(3)}$ [24]:

$$
\chi^{(3)}=\frac{n_{0} c}{7.91 \times 10^{2}} n_{2}+i \frac{n_{0}^{2} c \lambda}{\pi^{2}} \beta,
$$

where $\lambda$ represents the wavelength, $c$ is the speed of the light in vacuum, and $n_{0}$ the refractive index for low intensity (in esu units). When substituting in (7) the magnitudes for $\lambda=$ $355 \mathrm{~nm}, c=3 \times 10^{8} \mathrm{~m} / \mathrm{s}, n_{0}=1.42,\left|\chi^{(3)}\right|=1.05 \times 10^{-10} \mathrm{esu}$, and the $\beta$ value obtained by the single-beam transmittance experiments, the nonlinear refractive index was calculated. The nonlinear parameters are summarized in Table 1. It can 


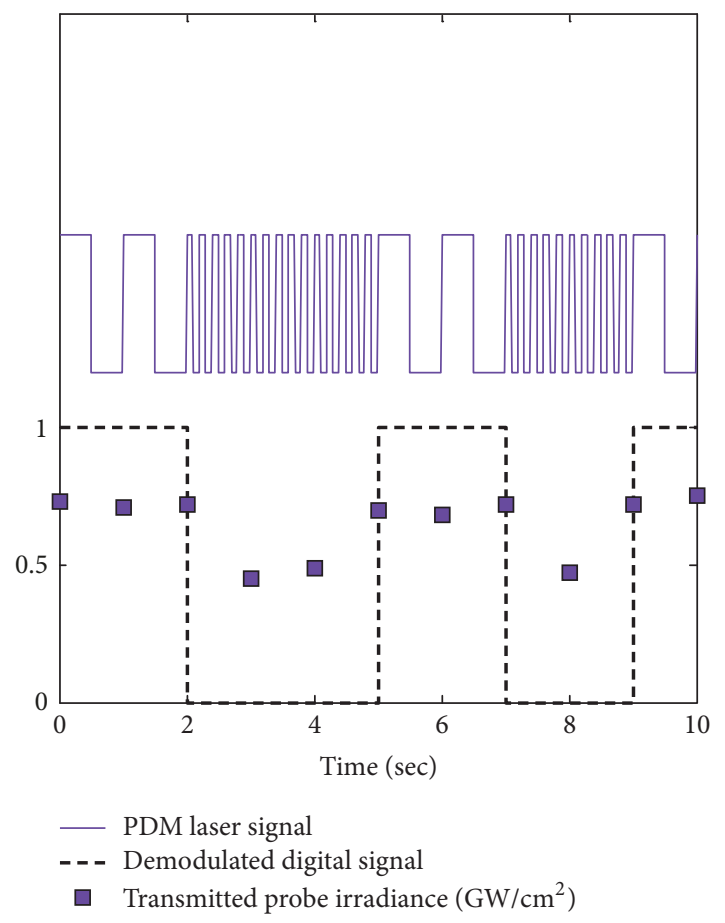

FIGURE 9: PDM signals demodulated by third-order nonlinear ultraviolet effects exhibited by SiQDs in silica. The irradiation was carried out by picosecond pulses at $355 \mathrm{~nm}$ and repetition rates of $1 \mathrm{~Hz}$ or $10 \mathrm{~Hz}$.

be considered that different contributions from free carriers, bound electrons, and thermal effects generate the strong differences in the femtosecond and picosecond response at the different wavelengths studied. Nonlinear optical effects are strongly dependent on shape and size of Si nanoparticles. Nevertheless, these results are in good agreement with previous investigations performed with silicon nanoparticles measured at different wavelengths [25].

Differences in nonlinear optical parameters described in Table 1 concern to irradiance, pulse duration, and wavelength variations for performing the experiments. These factors can be related to the dynamic response of electronic and thermal effects that should be present in the samples studied. The idea behind the fact that thermal effects play a key role in the difference comes from the numerical analysis and experimental observation of the changes in temperature illustrated in Figure 7. The intense nonlinear ultraviolet absorption properties exhibited by the SiQDs seem to be adequate to control discrete high-energy photonic interactions. In this respect, we consider the possibility of modifying the physical mechanism responsible for absorptive nonlinearity to modulate quantum processes in SiQDs by ultraviolet nonlinearities. It has been previously reported that two-photon absorption can be switched to saturated absorption in nanostructures by changing the wavelength that gives rise to the nonlinearity [26]. Moreover, it has been pointed out that the size, shape, and spatial distribution of nanoparticles can play a key role for the change of their characteristic nonlinear optical effects [27]. Figure 9 illustrates the nonlinear ultraviolet single-beam transmittance exhibited by the sample at two different repetition rates; here $f_{0}=10 \mathrm{~Hz}$ and $f_{1}=1 \mathrm{~Hz}$ represent binary PDM signals with $10 \mathrm{~Hz}$ describing the 0 logic value and $1 \mathrm{~Hz}$ corresponding to the 1 logic value. These plotted results (Figure 9) correspond to nonlinear demodulation based on SiQDs interacting with PDM signals in the proposed sample. From Figure 9 it can be seen that the sample acts like a low-pass filter for ultraviolet high-irradiance pulses. The difference between the magnitudes for the transmitted probe irradiances can be tailored by the synergy of different collections of SiQDs.

Considering that the temporal dynamics of energy transfer mechanisms can be employed for manipulating nonlinear transmittance processes, then the ability for demodulating PDM signals by nanostructures with ultrafast response can be contemplated. Important enhancement in refractive and absorptive nonlinearities was observed by infrared studies in comparison to ultraviolet results in our SiQDs. But remarkably, switching in the mechanism responsible for femtosecond absorption was observed in comparison to similar SiQDs measured by different input intensities [28].

Recent progress related to the development of nonlinear photonic systems with potential quantum functions has gained considerable attention by several research groups [29]. The possibility of manipulating low-dimensional signals for instrumentation and processing information is still a challenge that may take advantages of nanomaterials science and applied optics [30]. Encoding and ciphering processes constitute a clear example that advanced materials are currently required to improve the performance of their encrypting characteristics. Taking into account the opportunity to control all-optical interactions by nonlinear phenomena in SiQDs, diverse nanophotonic devices assisted by isolated nanoparticles can be envisioned. On the other hand, energy transfer based on SiQDs can play a fundamental role in applications that would be tuned by multiphotonic nonlinearities. In this work a clear indication about new possibilities for tailoring nanostructured samples to be used for ultrafast electromagnetic functions based on nonlinear excitations is highlighted.

\section{Conclusions}

Within this work the nonlinear infrared and ultraviolet properties exhibited by ion-implanted SiQDs in silica plates were analyzed. Strong absorptive and refractive nonlinearities effects were observed by ultrashort pulses at $830 \mathrm{~nm}$ and $355 \mathrm{~nm}$ wavelengths. The ability of the nanostructures studied for demodulating PDM signals by distinct near-resonance nonlinearities was pointed out. The increase in the pulse repetition rate of the laser system can result in the switch from saturated absorption to two-photon absorption in the sample, allowing attractive applications to be considered. This report emphasizes the need for attention of absorptive nonlinearities in controlled photothermal phenomena associated with silicon-based nanomaterials. The implications of these results may have the potential to develop low-dimensional all-optical modulating systems. 


\section{Conflicts of Interest}

The authors declare that there are no conflicts of interest regarding the publication of this paper.

\section{Acknowledgments}

The authors kindly acknowledge the financial support from CICESE, Instituto Politécnico Nacional, through Grant SIP20170498, Universidad Nacional Autónoma de México through Grants DGAPA-UNAM IN108217 and IN110216, and CONACyT through Grants 222485 and CB-2015-251201. The authors wish to thank K. Lopez and F. J. Jaimes for running the accelerator and J. G. Morales for his assistance during the sample preparation.

\section{References}

[1] Yu. Le, H. Han, W. H. Bin et al., "Complex hollow nanostructures: synthesis and energy-related applications," Advanced Materials, vol. 29, no. 15, pp. 1521-4095, 2017.

[2] P. Dong, Y. Chen, G. Duan, D. Neilson, and D. T. Neilson, "Silicon photonic devices and integrated circuits," Nanophotonics, vol. 3, pp. 215-228, 2014.

[3] D. Wang, L. Huo, Q. Wang, and C. Lou, "Performance optimization of ultra-short optical pulse generation based on Mamyshev reshaping and its application in $100-\mathrm{Gb} / \mathrm{s}$ and $200-\mathrm{Gb} / \mathrm{s}$ optical time-division multiplexing," Optics Communications, vol. 364, pp. 76-82, 2016.

[4] J. Leuthold, C. Koos, and W. Freude, "Nonlinear silicon photonics," Nature Photonics, vol. 4, no. 8, pp. 535-544, 2010.

[5] S. Isabelle and S. Jörg, "Metamaterial-inspired silicon nanophotonics," Nature Photonics, vol. 11, pp. 274-284, 2017.

[6] P. I. Borel, B. Bilenberg, L. H. Frandsen et al., "Imprinted siliconbased nanophotonics," Optics Express, vol. 15, no. 3, pp. 12611266, 2007.

[7] A. E. Krasnok, A. E. Miroshnichenko, P. A. Belov, and Y. S. Kivshar, "All-dielectric optical nanoantennas," Optics Express, vol. 20, no. 18, pp. 20599-20604, 2012.

[8] D. H. Spadoti, L. H. Gabrielli, C. B. Poitras, and M. Lipson, "Focusing light in a curved-space," Optics Express, vol. 18, no. 3, pp. 3181-3186, 2010.

[9] M. R. Shcherbakov, D. N. Neshev, B. Hopkins et al., "Enhanced third-harmonic generation in silicon nanoparticles driven by magnetic response," Nano Letters, vol. 14, no. 11, pp. 6488-6492, 2014.

[10] J. Bornacelli, J. A. Reyes Esqueda, L. Rodríguez Fernández, and A. Oliver, "Improving passivation process of Si nanocrystals embedded in SiOUsing Metal Ion Implantation," Journal of Nanotechnology, vol. 2013, Article ID 736478, 2013.

[11] S. Liu, G. A. Keeler, J. L. Reno, M. B. Sinclair, and I. Brener, "III-V Semiconductor Nanoresonators-A New Strategy for Passive, Active, and Nonlinear All-Dielectric Metamaterials," Advanced Optical Materials, vol. 4, no. 10, pp. 1457-1462, 2016.

[12] B. Shen, P. Wang, R. Polson, and R. Menon, "An integratednanophotonics polarization beamsplitter with $2.4 \times 2.4 \mu \mathrm{m}^{2}$ footprint," Nature Photonics, vol. 9, no. 6, pp. 378-382, 2015.

[13] P. J. Bock, P. Cheben, J. H. Schmid et al., "Subwavelength grating crossings for silicon wire waveguides," Optics Express, vol. 18, no. 15, pp. 16146-16155, 2010.
[14] M. R. Shcherbakov, P. P. Vabishchevich, A. S. Shorokhov et al., "Ultrafast all-optical switching with magnetic resonances in nonlinear dielectric nanostructures," Nano Lett, vol. 15, no. 10, pp. 6985-6990, 2015.

[15] P. Cheben, P. J. Bock, J. H. Schmid et al., "Refractive index engineering with subwavelength gratings for efficient microphotonic couplers and planar waveguide multiplexers," Optics Letters, vol. 35, no. 15, pp. 2526-2528, 2010.

[16] G. T. Reed, Silicon Photonics: The State of the Art, WileyInterscience, 2008.

[17] M. Sheik-Bahae, A. A. Said, T. H. Wei, D. J. Hagan, and E. W. van Stryland, "Sensitive measurement of optical nonlinearities using a single beam," IEEE Journal of Quantum Electronics, vol. 26, no. 4, pp. 760-769, 1990.

[18] C. Torres-Torres, A. López-Suárez, B. Can-Uc, R. Rangel-Rojo, L. Tamayo-Rivera, and A. Oliver, "Collective optical Kerr effect exhibited by an integrated configuration of silicon quantum dots and gold nanoparticles embedded in ion-implanted silica," Nanotechnology, vol. 26, no. 29, Article ID 295701, 2015.

[19] C. Torres-Torres, J. A. Reyes-Esqueda, J. C. Cheang-Wong, A. Crespo-Sosa, L. Rodríguez-Fernández, and A. Oliver, "Optical third-order nonlinearity by nanosecond and picosecond pulses in $\mathrm{Cu}$ nanoparticles in ion-implanted silica," Journal of Applied Physics, vol. 104, no. 1, Article ID 014306, 2008.

[20] J. F. Ziegler, J. P. Biersack, and M. D. Ziegler, SRIM - The Stopping and Range of Ions in Matter, Ion Implantation Press, 2008, http://www.srim.org.

[21] P. M. Fauchet, "Light emission from Si quantum dots," Materials Today, vol. 8, no. 1, pp. 26-33, 2005.

[22] Y. Qu, W. Ji, Y. Zheng, and J. Y. Ying, "Auger recombination and intraband absorption of two-photon-excited carriers in colloidal CdSe quantum dots," Applied Physics Letters, vol. 90, no. 13, Article ID 133112, 2007.

[23] F. Pevere, I. Sychugov, F. Sangghaleh, A. Fucikova, and J. Linnros, "Biexciton emission as a probe of auger recombination in individual silicon nanocrystals," The Journal of Physical Chemistry C, vol. 119, no. 13, pp. 7499-7505, 2015.

[24] R. W. Boyd, Nonlinear Optics, Academic Press, San Diego, Calif, USA, 1992.

[25] S. Hernández, P. Pellegrino, A. Martínez et al., "Linear and nonlinear optical properties of $\mathrm{Si}$ nanocrystals in $\mathrm{Si} \mathrm{O} 2$ deposited by plasma-enhanced chemical-vapor deposition," Journal of Applied Physics, vol. 103, no. 6, Article ID 064309, 2008.

[26] Q. Lin, O. J. Painter, and G. P. Agrawal, "Nonlinear optical phenomena in silicon waveguides: Modeling and applications," Optics Express, vol. 15, no. 25, pp. 16604-16644, 2007.

[27] K. Ikeda, R. E. Saperstein, N. Alic, and Y. Fainman, “Thermal and Kerr nonlinear properties of plasma-deposited silicon nitride/ silicon dioxide waveguides," Optics Express, vol. 16, no. 17, pp. 12987-12994, 2008.

[28] A. López-Suárez, C. Torres-Torres, B. Can-Uc, R. Rangel-Rojo, C. E. Valencia, and A. Oliver, "Third order nonlinear optical properties exhibited by a bilayer configuration of silver nanoparticles integrated to silicon nanocrystals embedded in ion-implanted silica," JOSA B-Optical Physics, vol. 32, no. 5, pp. 805-811, 2015.

[29] H. Chen, H. Liu, Z. Zhang, K. Hu, and X. Fang, "Nanostructured Photodetectors: From Ultraviolet to Terahertz," Advanced Materials, vol. 28, no. 3, pp. 403-433, 2016.

[30] D. Kufer and G. Konstantatos, "Photo-FETs: Phototransistors Enabled by 2D and 0D Nanomaterials," ACS Photonics, vol. 3, no. 12, pp. 2197-2210, 2016. 


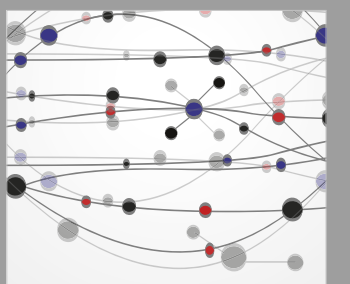

The Scientific World Journal
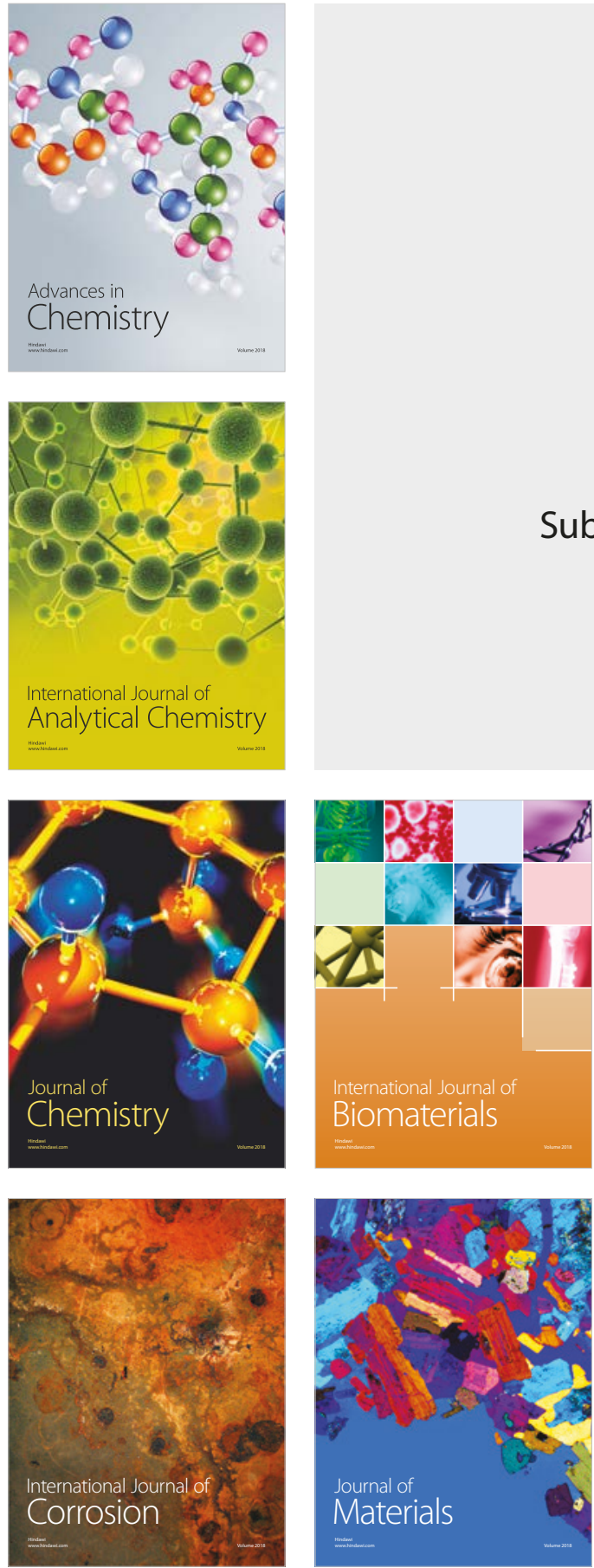

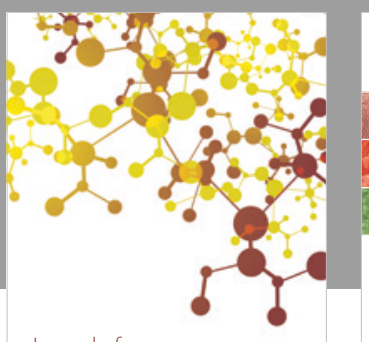

Journal of

Applied Chemistry
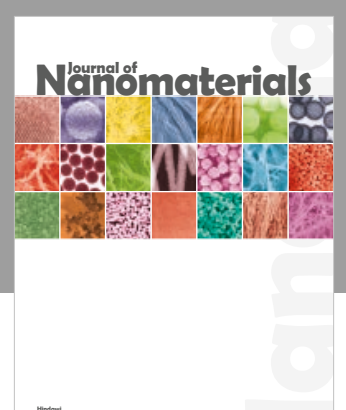

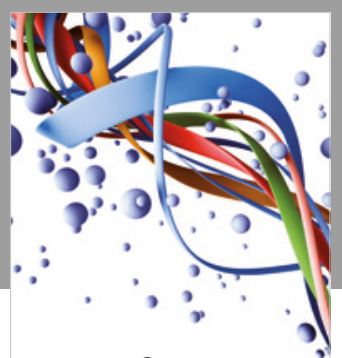

Scientifica

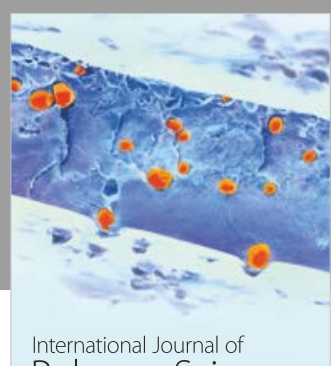

Polymer Science

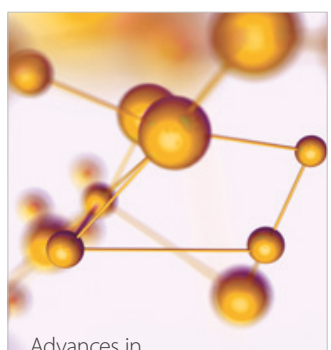

Physical Chemistry
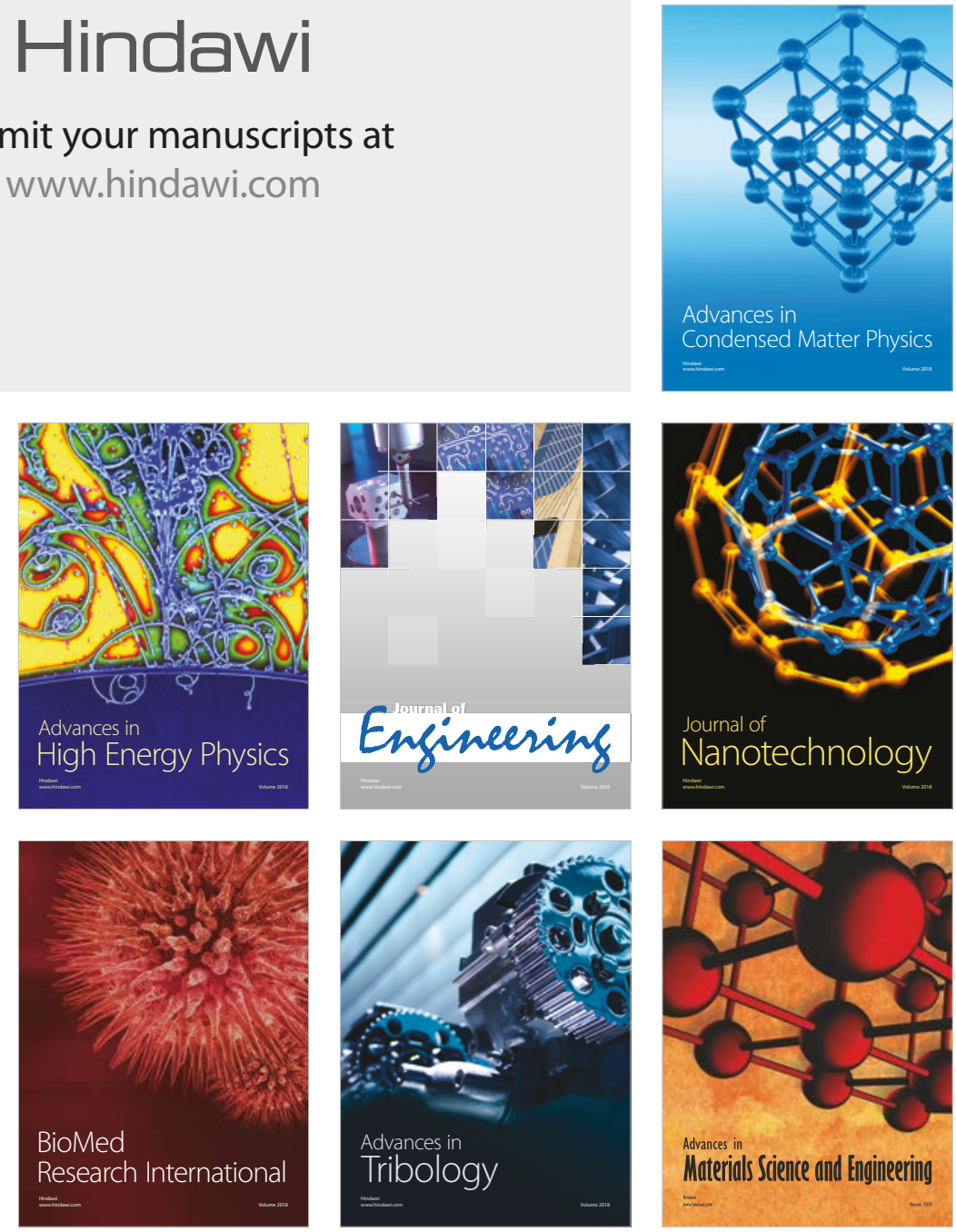